East African Medical Journal Vol.86 No. 4 April 2009

OUTCOMES OF ASSISTED REPRODUCTIVE TECHNOLOGIES AT THE NAIROBI IN VITRO FERTILISATION CENTRE

L. J. Noreh, MBChB, MMed (O/G), MSc. (Clinical Embryology), Clinical Director, Nairobi, IVF Centre, O. Tucs, BSc., MD (Molecular and Cell Biology), Clinical Embryologist, Nairobi IVF Centre, P.O. Box 29748-00202, Nairobi, Kenya, C.B. Sekadde-Kigondu, PhD, Associate Professor, Department of Clinical Chemistry, College of Health Sciences, University of Nairobi, P.O. Box 19676-00202, Nairobi, Kenya, and J.A. Noreh, RN, Dip. N, BSc. N, MSc. N, Nursing Director, Nairobi IVF Centre, P.O. Box 29748-00202, Nairobi, Kenya

Request for reprints to: Dr. L. J. Noreh, Nairobi IVF Centre, P.O. Box 29748-00202, Nairobi, Kenya

\title{
OUTCOMES OF ASSISTED REPRODUCTIVE TECHNOLOGIES AT THE NAIROBI IN VITRO FERTILISATION CENTRE
}

\author{
L. J. NOREH, O. TUCS, C.B. SEKADDE-KIGONDU and J.A. NOREH
}

\begin{abstract}
Background: Infertility is a common problem affecting up to ten per cent of married couples. A systematic evaluation of aetiologic factors forms the basis for choice of treatment and future fertility. On the global perspective, Assisted Reproductive Technologies (ART) has become internationally recognised treatment option for some infertile couples. A report on the current outcomes of ART practice at The Nairobi IVF Centre is presented.

Objective: To describe the practice of assisted reproduction and present a report on the current outcomes.

Design: A retrospective survey of data on assisted reproduction practice from August 2005 to July 2008.

Setting: The Nairobi IVF Centre a private medical practice in Nairobi, Kenya.

Interventions: Treatment of infertile couples by various assisted reproductive technologies including in vitro fertilisation (IVF), intra-cytoplasmic sperm injection (ICSI), uterine fresh embryo transfer (ET), and frozen/thawed uterine ET (FTET).

Main outcome measures: Fertilisation, embryo cleavage, ET, embryo freeze/thaw survival, preclinical pregnancy and clinical pregnancy rates, live births and other obstetric outcomes.

Results: A total of 362 IVF cycles were performed. Following controlled ovarian hyperstimulation, an average of 12 oocytes were retrieved per patient while the fertilisation and subsequent embryo cleavage rates were 67 and $91.2 \%$ respectively. An average of three embryos were transferred and the embryo transfer rate was $93.3 \%$ per cycle started and $96.6 \%$ per oocyte retrieval. Surplus embryos were available for cryopreservation in $106(50.1 \%)$ cycles. On thawing frozen embryos, the survival rate was $65.5 \%$. Following ET, the overall pre-clinical pregnancies were $124(36.2 \%)$ of which $99(28.9 \%)$ developed into clinical pregnancies per cycle. Of the clinical pregnancies, $72(73 \%)$ were singletons, $23(23 \%)$ twin gestations and four triplets $(4 \%)$. A total of 52 mothers have been delivered either by Caesarian section $45(86.5 \%)$ or spontaneous vertex delivery seven (13.5\%) to 61 live babies of whom 36 were females and 25 males. A further 28 IVF clinical pregnancies are ongoing, 26 following fresh embryo transfer and two from frozen/thawed embryos.

Conclusion: Our results on ART practice reflects a pregnancy outcome comparable to that reported by other fertility centres globally. Couples with infertility in East and Central Africa for whom ART treatment is indicated may now access the service locally.
\end{abstract}

\section{INTRODUCTION}

A couple is considered infertile if exposed to unprotected intercourse for one year or more without achieving a conception. On the global perspective, infertility affects up to ten per cent of married couples. A large proportion of these patients have a detectable lesion preventing conception and the most common aetiologic factor is tubal occlusion. An-ovulatory infertility, immunologic and other pelvic disorders are of lesser magnitude although male factors play a significant role $(1,2)$. The management of a couple with infertility requires an accurate initial diagnosis of the aetiologic factors and this forms the basis of prognostic evaluation for response to treatment and future fertility (3-5).

Following the pioneering work of Patrick Steptoe and Richard Edwards with the subsequent birth of Luice Brown in 1978, in vitro fertilisation (IVF) and embryo transfer (ET) has become internationally recognised treatment option for 
some infertile couples (6). In vitro fertilisation and ET is a stepwise process inclusive of controlled ovarian hyper-stimulation with pituitary gonodotrophins whichensuremultiplefolliculardevelopment where the recovery of more than one oocyte enhances the chances of achieving a pregnancy (7). The use of gonadotrophin releasing hormone analogues and lately antagonists is mandatory to pre-empt premature luteinising hormone (LH) surge and spontaneous ovulation $(8,9)$. Pre-ovulatory oocyte maturation is achieved by administration of human chorionic gonadotrophin (HCG) (10), and oocyte retrieval is carried out by transvaginal ultrasound guidance with or without anaesthesia. Fertilisation is aided by oocyte insemination with prepared and washed normal quality sperm (IVF) or intracytoplasmic sperm injection (ICSI) when sperm quality is sub-fertile. The subsequent culture in selected media, incubation levels of carbon dioxide and temperature is in fertilisation and embryo development. Pregnancy rates increase with the number of embryos replaced, up to three, above which pregnancy rates remain the same, attended with a higher order multiple gestations $(11,12)$. Currently, luteal support by a progesterone is preferred to HCG therapy.

Women with ovarian failure and men with sperm sub-fertility can achieve a pregnancy by utilising donor oocytes or donor sperm respectively. Oocyte and sperm donation programmes are subject to initial systematic counselling and health screening of those involved $(13,14)$.

Embryo freezing forms an integral part of routine IVF programmes worldwide. This reduces on wastage of valuable embryos, maximises the number of conception attempts per stimulation cycle and significantly increases cumulative pregnancy rates. The "freeze all" concept can be applied in various situations including the patient at risk of ovarian hyperstimulation syndrome (OHSS); asynchronous oocyte donor/ recipient cycles; endometrial unsuitability where implantation may be compromised and in failed fresh embryo transfer (15).

\section{MATERIALS AND METHODS}

The study is a retrospective survey of data on the treatment of infertile couples by ART at The Nairobi IVF Centre for the period starting August 2005 up to July 2008. Each couple's presenting clinical picture combined with a review of relevant medical investigative reports provided information on the underlying factors to their infertility. Couples with infertility in which ART was considered as the most appropriate option for treatment were advised accordingly. They were requested to undertake pre-treatment baseline work up inclusive of: Human Immunodeficiency Virus; Hepatitis B; Hepatitis C; Syphilis; relevant hormonal assays; pelvic ultrasound scanning and semen analysis on the male partner. A total of 362 IVF or ICSI and subsequent ET treatment cycles were performed.

Multi-follicular development was achieved by controlled ovarian stimulation with gonadotrophins together with gonadotrophin releasing hormone analogue or antagonist in one of the four pituitary down-regulation treatment protocols: long luteal; long follicular; short follicular or antagonist protocol as described by Elder and Brian (16) using either Suprefact (Aventis Pharma, Kent, UK) 500 micrograms as subcutaneous daily injection or Cetrotide (Serono, Bari, Italy) 250 micrograms as subcutaneous daily injection according to protocol specification. For ovarian stimulation, one of the three follicle stimulating hormones was administered as subcutaneous daily injection: Menopur (Ferring, Berkshire, UK); Gonal F (Serono, Bari, Italy) or Puregon (Organon, Netherlands) in varying doses ranging from 150 International Units (I.U.) to 450 I.U. according to the woman's age among other determining factors (16). Ovarian follicular and endometrial development was monitored by transvaginal ultrasonography. When the dominant follicle achieved a mean diameter of $18 \mathrm{~mm}$, pre-ovulatory oocyte maturation was facilitated by intra-muscular injection of Pregnyl (Organon, Netherlands) 10,000 I.U. approximately 34-36 hours prior to transvaginal ultrasound guided ovarian follicular needle aspiration. The latter was performed under $2 \%$ Lignocaine hydrochloride para-cervical block. Oocytes retrieved were identified under stereo microscopy, isolated and washed in syn vitro flushing medium (MediCult, Jyllinge, Denmark) and incubated in Universal IVF culture medium (MediCult, Jyllinge, Denmark) under 6\% carbon dioxide (BOC gases, Nairobi, Kenya) at $37^{\circ} \mathrm{C}$. After three to six hours incubation, the oocytes were either inseminated (IVF) or injected (ICSI) with prepared sperm as determined by assessed sperm quality. Male partners provided masturbatory semen which was prepared and washed by Suprasperm 80\% / 40\% discontinuous density gradients (MediCult, Jyllinge, Denmark), spin and swim up. For male partners with azoospermia, surgical sperm retrieval was achieved by testicular sperm aspiration (TESA). In cases where TESA could not realise sperm, and couples having had prior counselling, frozen donor sperm was sourced from a sperm bank for those who consented.

Signs of fertilisation were assessed 16-20 hours post insemination/ injection by inverted microscopy for presence of two pronuclei and 
two polar bodies. These pro-nucleate embryos were further cultured in Innovative Sequential Medium 1 (ISM I) (MediCult, Jyllinge, Denmark) up to day two post insemination when embryo cleavage and grading was done according to standard protocol (16). Up to three best grade embryos were selected, loaded into an embryo transfer catheter and procedurally transferred into the woman's uterus if the endometrial thickness $(\geq 8 \mathrm{~mm})$ was considered suitable for implantation (16). All surplus viable embryos were cryopreserved according the standard MediCult controlled rate freezing protocol. From the day of oocyte retrieval, luteal support on the woman was started using a progesterone, Cyclogest (Alpharma, Barnstaple, UK) $400 \mathrm{mg}$ pessaries inserted vaginally twice a day. On day 15 post ET, serum $\beta$-hCG bioassay was done and levels $\geq 20$ milli-international units $/ \mathrm{ml}$ was considered positive for pregnancy. Weekly rising serum $\beta$-hCG levels (chemical pregnancy) and ultrasonographic evaluation of a gestational sac, foetal poles and cardiac activity on day's 35-49 post ET confirmed clinical pregnancy. Progesterone was continued to complete ten weeks of luteal support, thereafter conventional antenatal care and subsequent delivery observed.

Frozen and thawed embryos were transferred either in a natural cycle or in a hormone replacement therapy cycle. ET in a natural cycle was possible if ovulation was detectable by monitoring pre-ovulatory LH surge and ovarian follicle tracking by ultrasonography to presumed ovulation. However, older women and those with an-ovular cycles were offered hormone replacement therapy for endometrial development as described by Serhd and Craft (13). Frozen embryos were thawed according to standard MediCult protocol and considered to have survived if $\geq 50 \%$ blastomeres at freezing had survived in the thawed embryos.

\section{RESULTS}

A total of 362 IVF treatment cycles are reported. The mean age for women and their male partners was 35 and 40 years respectively while age range was 20 to 50 for women and 24 to 77 for their male partners. Out of all patients who received IVF treatment, 281 had one cycle of ovarian stimulation followed by egg retrieval, fertilisation and ET; 31 couples returned for a second cycle; five couples had a third and one couple a fourth attempt. Thus 362 cycles were done for 318 couples or an average of 1.14 cycles per couple. Table 1 the aetiologic factors identified as cause of infertility for all couples who received IVF treatment at Nairobi IVF Centre. The causes of infertility were established by a comprehensive review of previous and current investigative medical records before the couple started IVF treatment.

\section{Table 1}

Aetiologic factors to infertility at Nairobi IVF Centre

\begin{tabular}{lccc}
\hline Aetiology of infertility & No. & $(\%)$ & $\%$ of Category \\
\hline Category & & & 58.0 \\
Female factors & 196 & 36.4 & \\
$\quad 123$ & & 10.7 & \\
$\quad$ Alocked tubes & 36 & 8.0 & \\
$\quad$ Menopause & 27 & 1.5 & \\
$\quad$ Premature ovarian failure & 5 & 0.9 & \\
$\quad$ Polycystic ovaries & 3 & 0.6 & 31.4 \\
$\quad$ Uterine disorders & 2 & & \\
$\quad$ Synachiae & 106 & 9.8 & \\
$\quad$ Hysterectomy & 33 & 4.7 & \\
Male factors & 3 & 0.9 & \\
$\quad$ Oligozoospermia & 24 & 7.1 & \\
$\quad$ Asthenozoospermia & 24 & 7.1 & \\
$\quad$ Teratozoospermia & 6 & 1.8 & \\
$\quad$ Oligoasthenoteratozoospermia & 33 & 9.8 & \\
$\quad$ Azoospermia & 3 & 0.9 & 0.9 \\
Other male causes & 338 & 100 & 100 \\
\hline Single women (donor sperm) & & & \\
\hline Totals $\quad$ & &
\end{tabular}


With regard to cause of infertility, female factors contributed to $58 \%$ while male sub-fertility was present in $31.4 \%$ couples. In 20 couples both male and female were diagnosed with infertility - related condition that brings total number of diagnoses to 338 in 318 couples. Blocked tubes $(36.4 \%)$ were the most common cause of infertility, followed by a $20.2 \%$ contribution from an-ovulation including menopause (10.7\%); premature ovarian failure $(8 \%)$ and polycystic ovaries $(1.5 \%)$. Thus, donated oocytes were utilised in $72(19.9 \%)$ cycles in which the woman was menopausal or had premature ovarian failure. In the two cases in which hysterectomy was the cause of infertility, the oocytes retrieved from ovarian stimulation were utilised in IVF surrogacy arrangement. Oligozoospermia $(9.8 \%)$ was the most frequent factor in male sub-fertility. Twenty four men $(7.1 \%)$ had azoospermia while in another 33 couples $(9.8 \%)$, the cause of infertility remained un-explained.

Controlled ovarian multi-follicular development was achieved by one of the analogue short follicular $(44.5 \%)$, long luteal $(23.3 \%)$, long follicular $(16.3 \%)$, or antagonist $(16.0 \%)$ pituitary down regulation protocols. The three varieties of follicle stimulating hormone (FSH) used for ovarian stimulation included menopur (59.3\%), Gonal F $(39.0 \%)$ or Puregon (1.7\%). The minimum and maximum daily dose of FSH was 150 and 450 I.U. respectively while the shortest and longest duration for ovarian stimulation was seven and 15 days. Ovarian follicular development was adequate in 341 cycles $(94.2 \%)$ while 21 cycles $(5.8 \%)$ were cancelled due to one of the following: inadequate ovarian response; endometrial insufficiency; failure of zygotes to cleave or no metaphase II oocytes available for ICSI.

At follicular aspiration, an average of 12 oocytes was retrieved. Most male partners 314 (90\%) sampled masturbatory semen while sperm was obtained by TESA in $16(6 \%)$ males mainly with azoospermia in addition to one where the male partner was unable to produce semen on the day of oocyte retrieval. Donated sperm from a sperm bank was used in $14(4 \%)$ treatment cycles in which the man had azoospermia (II), or the woman was single (3). Fertilisation was achieved by IVF in 150 cycles $(41.6 \%$ ) and ICSI in 210 cycles $(58.4 \%)$. The mean rate of fertilisation was $68.75 \%$, specifically $67 \%$ (1119 of 1669 oocytes inseminated) for IVF and $70.5 \%$ (1357 of 1925 oocytes injected) for the ICSI group. On the second day of embryo culture, $2259(91.2 \%)$ of the zygotes had cleaved to 2-8 cell embryos. Viable grade A, B and C embryos constituted $90.1 \%$ (2036 of2259 embryos) while the rest $(138,6.1 \%)$ were non-viable grade $D$ embryos. An average of three embryos was transferred to the uterus, but for various reasons, $<3>$ embryos per transfer was effected. The fresh ET rate was
93.3\% (338/362) per cycle started and $96.6 \%$ $(338 / 341)$ per oocyte retrieval. Surplus embryos were available for cryopreservation in $172(50.1 \%)$ cycles. Embryos in a further five cycles were all frozen without fresh ET due to either endometrial insufficiency (4) on would be day of ETor ovarian hyper-stimulation syndrome (1). In a total of 92 frozen thawed ET (FTET) cycles, 345 frozen embryos were thawed of which $226(65.5 \%)$ had survived the freeze/thaw process. FTET was effected mainly in hormone replacement therapy cycles in $87(94.6 \%)$ and the rest five $(5.4 \%)$ in a natural cycle.

Table 2 summarises pregnancy outcomes including both fresh ET and FTET cycles. There were $124(36.2 \%)$ cumulative pre-clinical pregnancies. Of these pre-clinical pregnancies, it was observed that $99(28.9 \%)$ developed into clinical pregnancies: 72 (73\%) were singletons; $23(23 \%)$ twin gestations and four triplets $(4 \%)$. Fourteen pregnancies $(15 \%)$ complicated in clinical abortions and five intrauterine foetal deaths (IUFD) (first twin IUFD with ongoing pregnancy and subsequent live birth of second twin (3); pregnancy induced hypertension, and nuchal cord asphyxia (1). There were three preterm deliveries, one due to pregnancy induced hypertension, while two triplet pregnancies complicated with premature rupture of membranes and premature labour respectively.

\section{Table 2}

Pregnancy outcomes following in vitro fertilisation and embryo transfer

\begin{tabular}{lcc}
\hline Pregnancy outcome & No. & $\begin{array}{c}\% \\
\text { per treatment } \\
\text { cycle }\end{array}$ \\
\hline Pre-clinical pregnancy & 124 & 36.2 \\
Clinical pregnancy & 99 & 28.9 \\
& $\%$ of clinical pregnancies \\
$\quad$ Singleton & 72 & 73.0 \\
Twin & 23 & 23.0 \\
$\quad$ Triplet & 4 & 4.0 \\
Pregnancy complications & $\%$ per treatment cycle \\
$\quad$ Clinical abortion & 15 & 15.0 \\
$\quad$ Ectopic pregnancy & 3 & 3.0 \\
$\quad$ Intrauterine foetal death & 5 & 5.0 \\
$\quad$ Preterm delivery & 3 & 3.0 \\
Live births (THBR) & 52 & 23.4 \\
Ongoing pregnancies & 28 & \\
\hline
\end{tabular}

THBR $=$ Take Home Baby Rate

There have been 52 live births, 42 resulting from fresh embryo transfer and ten from frozen thawed embryos. Singleton, twin and triplet births were 44 , seven and one respectively to 61 live babies. Forty five mothers $(86.5 \%)$ were delivered by Caesarian section and seven (13.5\%) had spontaneous vertex delivery to a total of 36 female and 25 male babies. Currently, there are 
28 ongoing clinical IVF pregnancies of which 26 resulted from fresh embryo transfer and two from frozen/thawed embryos.

There was no difference on pre-clinical and clinical pregnancy rates if couples came for a second IVF treatment attempt after the first failed. However none of couples carried pregnancy to term on the $3^{\text {rd }}$ or $4^{\text {th }}$ IVF attempt (Table 3)
$4 \%$ of the cycles. One important benefit of IVF or ICSI is the ability to observe the capacity of the oocyte to be fertilised, a process that puts to test both sperm function and oocyte quality. With the advent of ICSI as the standard approach to poor quality sperm, oocyte quality stands out as the primary factor to successful fertilisation, previously reported at $70 \%$ (5).

Table 3

Pregnancy outcomes following fresh ET in consecutive in vitro fertilisation cycles

\begin{tabular}{lccccc} 
Outcome & $1^{\text {st }}$ cycle & $2^{\text {nd }}$ cycle & $3^{\text {rd }}$ cycle & $4^{\text {th }}$ cycle & Total \\
\hline Pre-clinical pregnancy & 90 & 11 & 2 & 1 & 104 \\
& $(28.3 \%)$ & $(29.7 \%)$ & $(33.3 \%)$ & $(100 \%)$ & $(28.7 \%)$ \\
Clinical pregnancy & 64 & 9 & 1 & 0 & 74 \\
& $(20.1 \%)$ & $(24 \%)$ & $(16.7 \%)$ & & $(20.4 \%)$ \\
Live births & 34 & 8 & 0 & 0 & 42 \\
Ongoing pregnancies & 25 & 1 & 0 & 0 & 26 \\
\hline Total cycles & 318 & 31 & 6 & 1 & 362 \\
\hline
\end{tabular}

\section{DISCUSSION}

The results presented clearly demonstrate the realisation of conceptions and child births resulting from infertility treatment by ART at The Nairobi IVF Centre. On the global perspective millions of infertile women have conceived and progressed to child birth following treatment by ART.

The essential feature of controlled ovarian gonadotrophin stimulation is to achieve multifollicular development in order to enhance the potential for pregnancy in each treatment cycle. In our practice, transvaginal ultrasound guided follicular aspiration retrieved on average 12 oocytes per patient which is similar to that observed from other ART facilities (7-10). Semen analysis for sperm quality assessment forms the basis of evaluating aetiology of male infertility which in turn determines the selection of either IVF or ICSI for oocyte fertilisation (3-5). Fertilisation was achieved in $58.4 \%$ of our patients by ICSI and $41.6 \%$ by IVF. The higher prevalence of ICSI as a method of fertilisation is indicative of the significance of male factor infertility in our patients. Other prevailing factors do influence the choice of ICSI despite normal, sperm parameters. This is exemplified in the performance of ICSI in men with normospermia but low fertilisation rates in previous IVF treatment attempts and in cycles where fewer oocytes are retrieved. Couples with azoospermia will invariably require either surgical sperm retrieval by, TESA or utilisation of donor sperm. In our practice, TESA was performed in $6 \%$ and donor sperm utilised on
The fertilisation rate in our practice was $67 \%$ for IVF and $70.5 \%$ ICSI cycles. Pro-nucleate zygotes cleave to 2-8 cell stage on culture day 2, when the embryos are graded into four categories according to standard protocol (16). We observed embryo cleavage rate of $91.2 \%$ which compares well with other fertility centres of $92 \%$ (12). Grade A, B and C embryos are of good quality either for transfer to the uterus or cryopreservation. We achieved an embryo transfer rate of $93.3 \%$ per cycle started and $96.6 \%$ per egg retrieved and this is similar to reports from other units offering assisted reproduction $(11,12,16)$.

Cryopreservation of embryos is performed when there are surplus embryos after fresh embryo transfer. Surplus embryos were available for cryopreservation in $50.1 \%$ of the cycles. Other fertility units report a surplus in $16-88 \%$ of the treatment cycles and clinical pregnancy rate of $20 \%$ for FTET (16). Our clinical pregnancy for FTET was $15.2 \%$. In vitro fertilisation clinical pregnancy rates per cycle vary considerably, but the cumulative conception rates over a given number of treatment cycles compares favorably with natural conception. Our cumulative clinical pregnancy rate was $28.9 \%$ which compares well with that currently reported $(29.6 \%)$ for all IVF clinics in the United Kingdom (20). We report a multiple pregnancy rate of $27 \%$ (23 twin and four triplet pregnancies) which is comparable with $24.7 \%$ as reported by Helmerhest et al (17). In IVF/ET, multiple pregnancies are unavoidable but should be limited because most of the obstetric high risk factors are associated with it. A balance has to be achieved between the need to attain an 
acceptable pregnancy rate by the number of embryos transferred against the risk of multiple pregnancies.

Conceptions following assisted reproduction are high risk pregnancies and may be attended by obstetric complications. There is a higher rate of extra-uterine pregnancies after IVF due to thehigh prevalence of tubalfactorinfertility, with an estimated global prevalence of 4-8\% (18). We observed three tubal ectopic pregnancies in our practice. Soon following ET, utero-tubal myometrial activity may relocate embryos initially transferred to the endometrial cavity. The risk of spontaneous abortion following IVF is increased due to risk factors related to cause of infertility and not due to IVF procedure. This risk is increased independently whether they conceive spontaneously or by ART, suggesting genetic, chromosomal or implantation related problems (19). There were 15 clinical abortions in our series. It has also been observed that pregnancies following IVF/ET are associated with a high perinatal mortality by a factor of two when compared with the natural population, probably due to the higher prevalence of multiple pregnancies, preterm deliveries, ante partum haemorrhage and pregnancy induced hypertension that contribute to the larger number of low birth weight IVF babies $(16,17,20)$. We observed three still births, (two pregnancy induced hypertension and one nuchal cord asphyxia and three pre-term deliveries due to toxaemia, triplet pregnancy and premature rupture of membranes respectively. Due to various pregnancy and labour related factors, more than $50 \%$ IVF babies are delivered by Caesarean section. In our practice, we experienced exceptionally high Caesarean delivery rate of $86.5 \%$. As has been previously observed elsewhere, the male to female sex ratio of babies born approximates 1:1 (17), while our results show a skew of 26:35 respectively.

In conclusion, our interim results on ART practice reflects a pregnancy outcome comparable to that achieved by other fertility centres globally. We therefore recommend the utilisation of these services by infertile couples in whom ART treatment is indicated.

\section{REFERENCES}

1. The Eshre Capri workshop. Infertility revisited. The state of the art today and tomorrow. Hum. Reprod. 1996; 11: 1778-1807.

2. Mathews, T., Mati, J. K .G. and Fomulu, J. N. A study of infertility in Nairobi. East. Afr. Med. J. 1991; 58: 288-297.
3. World Health Organization. WHO Laboratory Manual for the Examination of Human Semen and Sperm-Cervical Mucus Interaction. 4th Edition. Cambridge: Cambridge University Press. 1999.

4. Rowe, P. J., Comhaire, F. H., Hargrave, T. B. and Mellows, H.J.WHOManual for theStandardization Investigation of the Infertile Couple. Cambridge. Cambridge University Press. 1997.

5. Hirsh, A. V. Male sub fertility. Br. Med. J. 2003; 327: 669-672.

6. Steptoe, P.C. and Edward, R.G. Birth after reimplantation of human embryo (letter) Lancet. 1984; 2: 1282-1285.

7. Ludwig, M., Felberbaun, R. E. and Diedrich, K. Ovarian stimulation from basic science to clinical applications. Reprod. Biomed. Online. 2002: 5: 73-86.

8. Hughes, E.G., Fedorko, D.M. and Daya, S. The routine use of gonadotrophin releasing hormone prior to in vitro fertilisation and gamete intrafallopian transfer: A meta-analysis of randomized controlled trials. Fertil. Steril. 1992; 58: 888-896.

9. Ai-Inany, H. and Abonglghar, M. GnRH antagonists in assisted reproduction: a Cochrane review. Hum. Reprod. 2001; 17: 874-885.

10. Drisoll, G.L., Tylo, J.P. and Hungan, J.T. A prospective randomized controlled, double blind, double dummy comparison of recombinant and urinary HCG for inducing oocyte maturation and follicular lutenization in ovarian stimulation. Hum. Reprod. 2000; 15: 1305-1310.

11. Cohen, J., Talansky, B. and Ali. K. Laboratory techniques for handling gametes and embryos. $\mathrm{Br}$. Med. Bull. 1990; 46: 643-653.

12. Mansor, R. and Abulghar, M.A. Optimising embryo transfer technique. Hum. Reprod. 2002; 17: 1149-1153.

13. Serhd, P.F. and Craft, I. L. Ovum donation-A simplified approach. Fertile. 1997; 48: 265-269.

14. Aird, I., Barrat, C. and Marsoch, A. BFS recommendation for good practice on screening of egg and embryo donors. Hum. Reprod. 1997; 3: 162-165.

15. Jones, H. W. Jr, Jones, D. and Kolm, P. Cryopreservation: a simplified method pf evaluation. Hum. Reprod. 1997; 12: 548-553.

16. Elder, K. and Brian, D. In vitro fertilisation. Second edition, Cambridge. United Kingdom. Cambridge University Press. 2000.

17. Helmerhorst, F.M., Perquin, D. A., Danker, D. and Keirse, M.J. Perinatal outcome of singletons and twins after assisted conception. A systematic review of controlled studies. Br. Med. J. 2004; 328: 261.

18. Schneider, J., Berger, C. J. and Catlel, C. Maternal mortality due to ectopic pregnancy. A review of 102 deaths. Obstet. GynecoI. 1997; 49: 557-561.

19. Wang, J. X., Nirrvan, R. J. and Wilcox, A. J. Incidence of spontaneous abortion among pregnancies produced by ART. Hum. Reprod. 2004; 19: 272-277.

20. Peter, R. Brindsen. A text book of in vitro fertilisation and reproduction. Third edition. Camb. UK. Taylor and Francis Publisher. 2005; 28: 489 - 497. 